\title{
Reflection on Online Teaching Practice During the Epidemic Period in Chinese Universities
}

\author{
Wei Jie*, Liu Xinxing \\ Yunnan University of Finance and Economics, School of Law, Kunming, China \\ Email address: \\ 15538060996@163.com (Wei Jie),20644426@qq.com (Liu Xinxing) \\ ${ }^{*}$ Corresponding author
}

To cite this article:

Wei Jie, Liu Xinxing. Reflection on Online Teaching Practice During the Epidemic Period in Chinese Universities. Science Innovation. Vol. 9, No. 5, 2021, pp. 194-199. doi: 10.11648/j.si.20210905.13

Received: September 7, 2021; Accepted: September 26, 2021; Published: September 30, 2021

\begin{abstract}
The Party and the country have promoted the establishment of a study-oriented society to a strategic height in the past six years, and the top-level design that is constantly strengthened has accumulated an institutional foundation for online teaching during the epidemic period. Moreover, the rapid development of MOOC lays a resource basis for online teaching during the epidemic period. The changes in both online teaching situations and communication means bring about innovation in teaching ideas and teaching methods, and teachers must become a main force of the innovation. Meanwhile, they should learn a lesson from traditional education and MOOC, and focus on "collaborative learning" to perform teaching design and innovation. The smooth going of online teaching this time proves that grid management mechanism in universities is remarkably efficient. However, as for the quality supervision of online teaching, universities should continue to explore more scientific and more reasonable quality evaluation index systems and evaluation procedures, more reasonable performance-based pay calculation methods and honor allocation systems, more sufficient financial support for teaching and research, and even title promotion conditions that center on teaching rather than scientific research, so as to motivate course teachers to throw themselves into teaching design and innovation persistently.
\end{abstract}

Keywords: Online Teaching, Collaborative Learning, Grid Management Mechanism, Quality Supervision, Incentive Strategy

\section{中国高校疫期在线教学实践反思}

\section{魏杰”, 刘新星}

云南财经大学法学院, 昆明, 中国

\section{邮箱}

15538060996@163.com（魏杰），20644426@qq.com（刘新星）

\begin{abstract}
摘要：过去六年, 党和国家将建设学习型社会提到战略高度, 不断加强的顶层设计为疫期在线教学积累了制度基础; 慕课的快速发展为疫期在线教学奠定了资源基础。线上教学情境的改变与联通手段的改变带来了教学理念与教学方法 的革新, 教师必须成为革新的主力, 同时汲取传统教育与慕课的经验、教训, 围绕“协作学习”进行教学设计与创新。 此次在线教学能有条不紊地推展开, 网格化的高校管理机制被证明成效卓著; 但对在线教学质量的监督, 仍有赖于各 高校持续探索更科学、更合理的质量评估指标体系与评价程序; 更合理的绩效计算方法和荣誉分配办法, 更充足的教 研经费支持，甚至偏向教学而非科研的职称晋升条件，将激励任课教师在教学设计与创新中持续投入。
\end{abstract}

关键词: 在线教学, 协作学习, 网格化管理机制, 质量监督, 激励策略 


\section{1. 引言}

2020年1月27日, 教育部先发布了《关于 2020 年春季 学期延期开学的通知》, 后于 2 月 4 日又发布了《关于在疫 情期间做好高校在线教学组织与管理工作的指导意见》

(教高厅 (2020) 2号)，向全国各高校提出“停课不停教、 停课不停学”的总体要求, 要求各高校立即制定在线教学 组织与实施方案, 以保证线上、线下教学质量实质等效为 前提, 推进教学改革创新, 完成教学任务。为了使各高校 都能很快展开线上教学, 教育部在 2 月 2 日已组织 22 个在线 课程平台免费开放 2.4 万余门专业门类齐全的高质量线上 课程, 同时全天候开放国家虚拟仿真实验教学项目共享平 台 (实验空间), 免费提供2000余门虚拟仿真实验课程资 源。截止4月10日，教育部高等教育司主办的“中国高校在 线教学国际平台课程建设工作视频会议”在北京召开, 共 有 37 个基础好、实力强的课程平台和技术平台支持全国高 校在线教学, 另有 110 余家社会和高校平台也参与其中, 免费开放慕课和虚拟仿真实验等在线课程已达 4.1 万门, 涵盖本科 12 个学科门类, 专科高职 18 个专业大类。

至2月17日, 各地高校陆续“网上开学”。截止4月3日, 全国高校学生在线学习人次达到 11.8 亿; 据 37 个专业在线 课程平台统计数据显示，2月17日-3月30日间的日活平均 人数达到 4939.1785 万。

疫期对网络教学的迫切需求推动了线上教学与线下 教育的更进一步衔接。粗浅的看, 线上教学只是为线下教 育提供了一种技术性的新手段。然而, 教学情境的转换直 接改变了教与学的关系, 也颠覆了传统教学理念。线上教 学与线下教学在学习体验、教学方式与手段、教学目标等 方面都存在差异, 如何才能使两者真正融合, 并着实提高 教学质量, 这给学校与教师提出了很大挑战。为此, 本文 将对在线教学的本质进行探讨、对疫期在线教学实践情况 进行分析, 揭示在线教学存在的问题与面临的挑战, 并为 线上线下教学的真正融合提供策略参考。

\section{2. 疫期在线教学之运行基础: 平台与资源}

在线教学对于中国高校而言并非全新事物, 而是远程 教育的新一代发展。中国自19世纪50年代兴起的“函授教 育” (成人教育) 借助邮递系统与邮寄印刷学习资料, 形 成了以“自学为主, 面授为辅; 通信答疑, 在场考试”的第 一代远程教育。19世纪80年代, 随着广播电视、录音录像 技术、计算机技术的发展而兴起的“广播电视教育”形成了 第二代远程教育, 中央电视大学的成立与发展是第二代远 程教育的代表。随着互联网、大数据、人工智能等现代信 息技术的迅猛发展, 以慕课为代表的网络课程成为第三代 远程教育之代表。此次疫情发生之前, 在线教学主要体现 为慕课。

2013年中国慕课开始起步, 清华大学发起建立国内第 一个慕课平台——“学堂在线”, 目前已汇集全球 600 余所 知名院校课程。2014年, 网易公司与高等教育出版社合作 开发出“中国大学MOOC”平台, 汇聚了教育部评定的国家 精品课程以及众多知名高校优质课程, 已成为国内课程数
量最多的慕课平台。除此之外, “好大学在线”、“育网开 放教育平台”、“优课联盟”等都是国内有影响力的慕课平 台。据2019年中国慕课大会发表的《中国慕课行动宣言》, 十余个国内高校和机构自主研发的平台共上线12500门课 程, 超过 2 亿人次在校大学生和社会学习者参与学习, 6500 万人次大学生获得慕课学分。据《教育部关于加快建设高 水平本科教育全面提高人才培养能力的意见》（教高 [2018]2号)：建立学分认定制度、推动优质课程资源开放 共享、促进慕课等优质资源平台发展是中国慕课未来发展 的重点任务。

过去六年, 党和国家将建设学习型社会提到战略高度, 不断加强的顶层设计为疫期在线教学积累了制度基础; 慕 课的快速发展为疫期在线教学奠定了资源基础——除了 为所有高校提供了随手可用的平台、技术和课程资源, 也 带动了高校内部网络课程建设; 新的线上教育理念也在慕 课推广过程中被更多教学工作者认识、实践与研究。据全 国高等学校质量保障机构联盟 (CIQA) 的调查: [2]在参 与调查的334所高校所涉 13997 名教师中, $81.65 \%$ 的教师此 前接受过线上教学培训, $20.43 \%$ 的教师在疫情之前开展过 线上教学, 仅有 $2.38 \%$ 的教师不能熟练使用平台技术。正 是因为有基础, 自教育部发布在线教学通知与要求之后, 各高校才能有条不紊地制定在线教学组织与实施方案, 对 时间安排、课程范围、教学准备相关工作、教学运行具体 部署、组织保障等问题都进行了因地制宜地的规定, 保障 了在线教学平稳运行, 真正实现了“停课不停教、停课不 停学”。

\section{3. 疫期在线教学之根本问题: 如何“保质”}

虽然慕课与疫期在线教学本质上都是远程教育, 但疫 期在线教学与其他远程教育的立足点明显不同。正如英国 爱丁堡大学数字教育研究中心杰里米. 诺克斯博士所言, 慕课自表明致力于解决高等教育机会不平等之问题后, 才 得到大规模推广; 慕课的愿景主要面向那些因地理、经济 原因缺乏受教育机会的人群; 大规模招生与开放获取资源 使人们一度将其看作一场革命。[3]亚洲各国的慕课大多免 费开放, 其定位与目标主要是实现国内高等教育公平、促 进平等就业。[4]《中国慕课行动宣言》也提出: “中国慕 课要把推进高等教育区域和校际公平作为基本价值取向”。 事实上, 不论最初的函授教育或是之后的广播电视教育, 乃至慕课都倡导教育资源的平等、开放、共享。然而, 这 些都不是疫期在线教学所追求的——“保证疫情防控期间 教学进度和教学质量”才是其初衰。当然, 保质势必要求 技术跟进、教学创新, 但创新并非根本, “保质”乃第一要 务。

\section{1. 远程教育存在的共性问题与对策}

远程教育的特点在于重建时空, 将分离的“教”与“学” 透过技术重新实现连接互动, 实现教育资源开放共享, 大 规模学习者跨时空参与的交互学习模式。然而, 正是因为 “教”与“学”的不同步使远程教育的教学体验与线下教育 
截然不同——如何实现“教”与“学”的再度整合, 增强学习 体验, 保证教学效果与质量, 这正是围绕远程教育一直被 探讨的议题。[5]对于疫情防控期间的各高校来说, 将线下 教学全部迁移线上实乃情势所逼, 但所有高校都必须积极 面对“如何保证教学效果与质量”这一考题并给出令人满 意的答案。

据“高校教学信息化与教学方法创新教学指导委员会” 总结: 慕课、直播、录播、远程指导是疫期在线教学四种 基本形态; $1+\mathrm{M}+\mathrm{N}$ 协同跨校教学模式、MOOC $+\mathrm{spoc}+$ 线上 翻转课堂、 $\mathrm{SPOC}+$ 线上翻转课堂、视频公开课/资源共享 课/线上指导、在线教学工具 + 会议系统、教学材料共享+ 线上指导是六种典型模式。[6]据CIQA的调查报告, 经常 使用MOOC和录播的受调查者均未到 $30 \%$, 直播+远程指 导更受青崃。究其原因, 仍然和学习体验、教学效果相关。

慕课发展经历了CMOOCS和XMOOCS两个阶段。如 今, 国内外主要慕课平台所提供的课程资源基本都属于 XMOOCS学习管理型。[7]学习管理型慕课背后的支撑是 认知主义学习理论而非构建主义学习理论。认知主义学习 理论把大脑看作一台电脑, 教学过程体现为“传输、刺激大脑-反应”: 教学就是向大脑传输外部真理, 学生的任务 在于准确复述所学内容; 数量比质量更重要, 传输什么与 怎么传输是关键; 如果技术传输更有效率，教师完全可以 被取代; 因而, 学习也不过是一种个人体验和事件。 [8]Coursera平台创办者Andrew Ng也承认: “与上大学相比, 参加慕课学习仍然是一个更加孤独的经历。”[9]授课视频 发布、学习进度控制、作业通关反馈、学习同伴互评、教 师论坛答疑是慕课结构化的学习流程——看视频、做作业、 等反馈, 并未实现预想的灵活、自适应、个性化的学习方 式, 反而造成了课程学习互动性不强、参与度不高、体验 性差; [10]辍学率高、持续性不强 [11] 一一份关于 Coursera平台上宾夕法尼亚大学慕课学习者的研究也显示: 在课程开始 2 周后, 学生的在线活跃度与参加测验人数都 急剧下降, 坚持到课程结束的人非常少。[12]

鉴于上述问题, 强调社会学习环境的建构主义学习理 论与在线协作学习模式被倡导融入慕课。建构主义学习理 论认为: 意义和理解源于社会交互, 人与人之间的讨论与 互动在学习过程中是首要的; 对于学习和人的发展而言, 社会环境非常重要。在线协作学习模式强调: 学生在教师 帮助下开展讨论和协作 (研讨、辩论、角色扮演、小组项 目, 学术性、科学性和专业性讨论) 是教与学之关键, 而 不是辅助手段; 应当根据讨论和协作活动选配课程资源 (读物、视频或教科书)，而不是反之; 技术能促进学生 讨论、协作及师生互动, 但无法替代这些活动。[8] ursera 平台另一位创办者Daphne Koller和Udacity首席执行官 Sebastian Thrun也承认, 慕课应当融入在线协作学习模式。 为此, 融入了线上线下混合教学模式的 SPOC和翻转课堂 被开发出来, 既体现了MOOC的优点弥补了线下教学的不 足, 又融入了线下教学的优点弥补了MOOC的不足。美国 斯坦福Udacity慕课平台在 2017 年启动了为期三个月的 Udacity Connect项目, 即要求学习者在周末参加线下实体 课堂, 接受五个小时面对面学习指导和项目任务, 学习完 成率比单纯在线学习提高了 5 倍。[13]

\section{2. 疫期在线教学存在的问题与对策}

据CIQA调查报告, 对线上教学有明确好感体验的学 生略微超过 $50 \%$; 但认为线上教学效果比传统教学效果差 的学生人数比持相反意见的人数多 $7.7 \%$; 认为线上教学效 果高于线下教学效果的教师人数与持相反意见的人数持 平。如何理解这一结果呢? 事实上, 不论录播还是直播, 如果教师只是对着摄像头如在教室里一样讲授, 那么, 脱 离了面对面的眼神交流、肢体语言沟通、群体学习效应及 在场监督效应, 教学效果可能无法比拟现场。此时, 如何 设计与开展在线协作学习, 就成为每位任课教师必须充分 认识并深入思考的问题。同样, 据该调查报告, 不论教师 同时选择直播、录播、慕课还是文字+音频的讲授方式, 线上互动研讨、提供材料供学生自学都是疫期在线教学常 规方式; 每门课程平均使用 2.16 个教学平台也说明, 教师 们正在使用功能不同的平台应对讲授与互动这两大环节 的不同需要。据调查, 教师们认为学生自主学习能力是影 响在线教学效果的首位因素, 学生自主学习能力弱也被认 为是排在前几位的在线教学所存在的问题之一。然而, 这 难道不也是过去教师们对线下教学的看法吗。

问题的关键在于, 线上教学不仅仅是教学情境的改变 与联通手段的改变, 正是由于教学情境的改变与联通手段 的改变直接带来了教学理念与教学方法的革新, 教师必须 成为革新的主力。疫期线上教学必须同时汲取传统教育与 慕课的经验、教训, 围绕“协作学习”进行教学设计与创新。 正如有 100 多年远程教育历史的英国开放大学学者吉利・ 西蒙所言, “网络技术为人们提供了一种获取世界信息的 新窗口, 也可能会降低学习者和学习活动的社会化程度及 协作水平”, “要建设一个富有创造性的时空......而不是将 原来的课程照搬到在线课程中”。[14]美国 QM(Quality Matters)项目研发的《网络学习质量指南》也指出, “归根 到底, 网络课程的质量依赖于教师课程设计的质量以及教 师对教学方法的适用。”[15]

如何围绕“协作学习”进行教学设计与创新, 这是富有 挑战但并非无迹可寻的难题。英国开放大学的在线课程多 为“故事讲述型”: 强调以故事为中心创设学习环境, 刺激 学习者的好奇心; 以学习任务的形式要求学习者提出科学 问题、设计实验、进行预测、提出并验证假设, 引导学习 者进行探索学习; 有效发起学习讨论, 使学生可以与教师、 同学分享学习经验与不同观点, 维持有效的学习参与 [16] ——提升学习者体验, 为学习者提供可用性高的社会 性学习体验环境, 这是英国开放大学的成功经验。建设网 络学习社区则是实现“协作学习”的另一种策略。美国QM 的《网络学习质量指南》指出, 通过社交网络系统创建非 实时在线讨论、聊天室、协作项目和学习小组是这种策略 的主要路径, 教师的作用贯穿其中是质量保证: 规划组织、 示范有效网上行为、对学生进行单独指导与鼓励、创建小 组、随时在线、担任讲师角色、沟通者角色——都是教师 的任务。[17]

在由中国国家标准化管理委员会与国家市场监督管 理总局联合发布, 2019年4月1日实施的《信息技术、学习、 教育和培训在线课程国家标准》（GB/T36642-2018）中: 学习者控制 (即全面支持学习者按照自己需要对学习环境 
进行个性化定制），内容交互性（课程提供充分的交互机 会），交流与协作（课程内容中充分包含学习者讨论或协 作解决的问题及相应要求), 动机兴趣 (采用适当策略吸 引学习者的注意力, 且策略与课程内容紧密结合, 吸引注 意力效果好), 实例与演示 (在需要深刻理解和灵活应用 的知识点上尽可能提供多种不同实例和演示, 反映知识在 不同情境中的变化）、学习帮助（提供适应具体学习内容 和学习环境的学习指导与帮助) 、练习反馈 (提供有助于 学习者改正错误和增进理解的判断信息) 都是评价在线课 程内容设计的重要指标。这些指标也都充分体现出个性化 学习、协作学习特点。虽然该国家标准仅指向由在线学习 平台承载和运行的课程, 但该标准无疑也给各高校的任课 教师建立了明确参照。

教育部发布的《普通高等学校本科教学工作合格评估 指标体系》 (教高厅[2011]2号) 对线下课程内容设计的评 价是非常粗略的, 各高校也都有自己的教学质量评估规定, 但关于教学内容组织与教学方法手段的评价指标仍然比 较笼统, 也不是围绕交互协作型学习模式而设立, 主要强 调知识的体系性、理论结合实践的教学方式。可见, 在保 证质量的基点上, 疫期在线教学对所有任课教师提出了全 新要求, 相当于要求所有任课教师经历“一场头脑风暴”, 打破线下教学的固化思路, 重组新的教学模块, 重新建立 自己与学生之间的教学交互模式。也许应当将其看作一种 机遇。当同时掌握线上教学理论方法与多媒体传输技术, 不断探索如何将两者糅合并融入教学全过程, 教师将逐步 体悟到: 借助技术的记忆、再现、监测、反馈、大数据分 析, 在课堂上所能做的其实比传统教学更多, 所有关于教 学的奇思妙想可能都会实现。

\section{4. 疫期在线教学之运行保障: 管理、监督与激 励}

如果说面对突如其来的疫期教学, 高校教师必须思索 的问题是: 如何认识与接受新教学理念? 如何创新教学手 段? 如何重建师生交互模式? 如何运用新技术? 那么, 高 校应当思索的问题是: 如何更新管理模式? 如何评估教学 质量? 为教师提供什么支持与激励?

\section{1. 疫期在线教学的管理支撑}

此次在线教学能有条不紊地推展开, 首先是教育行政 系统内部自上而下统一认识、命令贯穿; 其次是各级教育 行政部门负责各辖区的组织、协调和指导工作; 最后是各 高校、各种课程资源平台的积极响应、执行参与。甚至在 高校内部也自有一套极具执行力的响应机制。自教育部发 布线上教学要求起, 在不到半个月的时间里, 各高校从在 线教学方案部署到方案顺利执行, 联动起来的不仅有高校 行政管理系统、教务管理系统、教学辅助系统 (网络技术、 图书馆资源）、后勤系统, 还有教师、学生、甚至家长。 事实证明, 这样一种网格化的管理机制（如图1）在高校 应对突发公共卫生事件的行动过程中成效卓著。

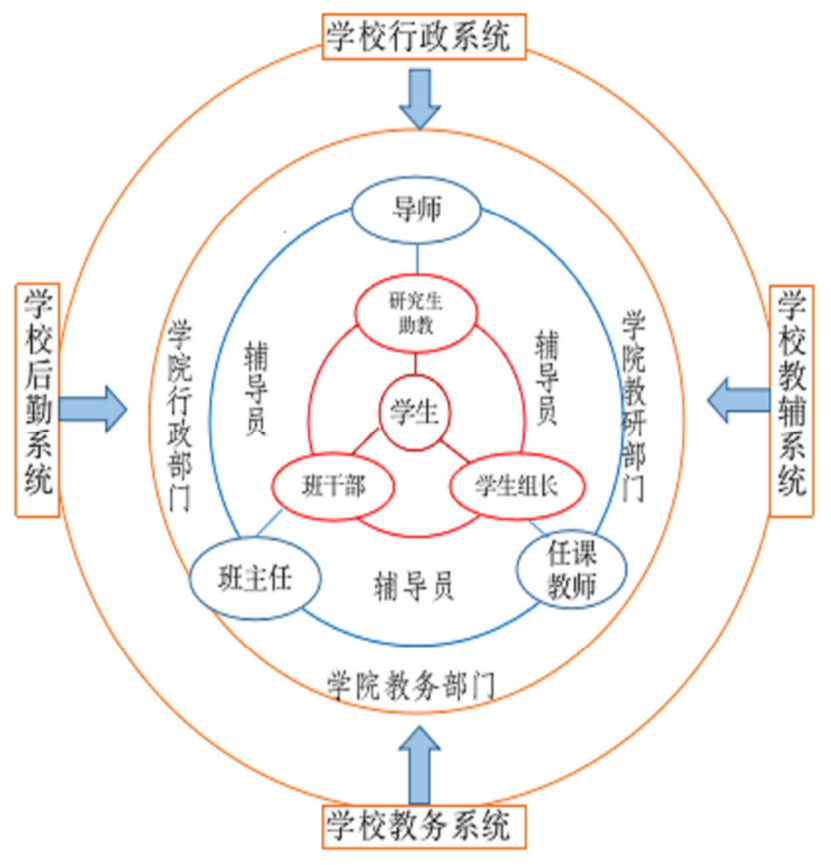

图1 高校网格化的管理机制。

虽然人数众多的学生分散在大江南北不同角落, 但通 过学生之间的横向连接机制, 以及学生与教师之间的纵向 连接机制（包括教师与家长之间的联络机制），教师得以 充分掌握学生的健康情况、学习情况和生活情况: 既能及 时获取需要帮助的学生信息, 也能实时督促学生按时到课、 整改学习纪律。而教师所属学院的纵向党政组织系统与横 向教务、教研组织系统也使学院得以充分掌握每位教师的 相关情况, 能有效组织教师——通过教师组织学生, 保持 疫期在线教学正常运转。疫期在线教学所需要的设备技术、 数据资源、网络维护等都不是某一个学院足以提供的, 因 而, 学校除了向学院输出行政命令与政策决议以外, 还必 须提供: 更优质的网络环境, 如网络通畅快捷; 更稳定的 设备软件, 如不卡顿、不经常掉线的直播软件; 更强大的 数据平台, 如能完整记录学生、教师的教学行为数据; 更 及时的资源技术服务, 如实时监控技术故障、给教师一对 一的技术帮助、帮助教师搜寻传递学术资源。事实上, 这 几个方面的支持也意味着学校必须把部份的行政管理工 作、部份的教务管理工作、教辅工作都迁移到了线上。这 对于各高校来说像是一次检视与演习; 对部分信息化建设 相对落后的高校而言, 未来是否值得在信息化建设方面增 加投入, 相信答案已经很明确。

\section{2. 疫期在线教学的质量监督}

如果说, 如何创造更好地教学效果、保证教学质量是 困扰教师的主要问题, 那么, 如何评估线上教学质量也相 对成为学校或学院的难题。

传统教学评价与线上教学评价的共同之处在于, 都结 合了结果评价与过程评价。结果评价主要通过考查学生对 知识的掌握程度与水平来反映教学质量; 过程评价则通过 考察学生的学习过程来反映教师的教学水平。在线下教学 过程中, 测验、考试是结果评价的主要手段; 同行评价、 
教学督导评价、学生评价是过程评价的主要手段。线上教 学更强调学生主动参与, 师生互动、协作, 因此, 教师对 互动环节的设置和指导行为, 教师与学生参与互动的频率, 学生的学习时长、活动记录、学习成果与作品, 也被纳入 评价范畴。然而, 传统教学的评价过程常常被质疑过于“分 数化”, 在线教学的评价过程也被质疑容易陷入“数字化”。 [18]问题在于: 在线下教学的评价过程中, 学生的结课总 成绩与教学过程评价结果并没有很好地结合起来, 二者之 间的影响关系在评价过程中并未得到合理考量; 而在线上 教学的评价过程中, 目前的评价手段是否足以充分衡量学 生能力的提升与思维的发展, 还未有定论。

在线教学运行2-4周后, 辽宁、福建、黑龙江、陕西、 天津、北京等省份教育厅陆续发布了“在线教学运行报告” 或“质量报告”。浙江大学、清华大学、南京医科大学、北 京交通大学等是较早对外发布报告的高校。这些报告与 CIQA的调查报告一样, 都主要是对在线教学的运行情况 (如课程开设数量、专业开课分布、开课平台使用情况、 学生人数、技术服务情况等），以及师生对平台、技术服 务保障的满意度, 学生对教学环节、教学效果的总体评价, 教师对教学效果的自我评价与对学生学习情况的总体评 价。承前所述, 师生对教学环节、教学效果的满意度和看 法仅属于教学评价的一个方面, 并不能完全反映出在线教 学质量。任课教师在教学软件后台所能看到的学习行为记 录仍然是有限的, 主要包括出勤、在线时长、作业完成情 况, 互动参与仅体现为弹幕发送次数或讨论区发言次数。 关键是, 被化约为数据的学习行为在评价过程中是否还能 被还原展现, 这就是所谓的“数字化”问题。对于教师互动 环节设计、课程内容安排、指导参与等方面的评价, 仍有 赖于各高校制定科学的评价指标与评价程序。这项工作在 疫期在线教学阶段可能无法完成, 但对于各高校而言, 即 使在疫情过去之后, 持续探索更科学、更合理的质量评估 指标体系与评价程序, 不仅是应对未来智能化教育改革之 需, 也能促进线下传统教学评价机制的完善。

\section{3. 疫期在线教学的激励策略}

在慕课大规模推广的过程中, 线下课堂是否会被完全 取代曾引起热议, 甚至有人担心人工智能将使大批教师失 业。如果教学仅指课堂“灌输式”讲授配合测验, 那么, 答 案是肯定的, 人工智能比真人教学更有效率。然而, 远程 教育所存在的问题已经揭示教师的作用无可替代。远程教 育所指向的机会公平与资源共享, 早就蕴含在中国两千多 年前“有教无类”[19]的教育理念中，现代信息技术革命确 实使之成为可能; 协作型学习也可以看作是“教学相长”的 现代实践; 然而, “因材施教”[21]却是任何类型的远程教 育都无法实现的。任何富有成效的学习都需要教师针对学 习环境、学习对象、学习内容做不同设计, 也需要教师真 正参与到学习者的学习过程中去。

根据CIQA关于“学生对线上教学优缺点评价”的调查 结果, 优点主要体现在名师名课得到共享、学习自主选择 余地更大等方面, 而缺点主要体现在教师无法充分掌握学 生学习情况、交流互动受限等方面。而根据“教师线上教 学所遇到的最主要困难”和“所面临的最大挑战”这两项调
查, 课堂组织、交流、互动是最主要的困难, 需要改变教 学策略和方法, 以及改变以往的教学观念、习惯则是最大 挑战。

一门成功的慕课, 从开发、上线到运行完善至少需要 花2-3年的时间、几十万到几百万不等的投入, 以及组建 包括教师、教研专家、技术专家、评估专家等在内的专业 团队。可见, 对于在线教学任课教师来说, 要获得较好地 教学效果必须数倍投入时间、精力。对此, 学校除了能够 给予设备、网络、技术等方面的支持之外, 科学的评估体 系与政策激励更为重要。要求所有任课教师的在线教学效 果能比拟成功的慕课是极不现实的, 但学校可以通过更合 理的绩效计算方法和荣誉分配办法, 更充足的教研经费支 持, 甚至偏向教学而非科研的职称晋升条件, 激励任课教 师在教学设计与创新中持续投入。

\section{5. 结论}

随着国内疫情基本得到控制, 自2020年4月10开始各 地高校进入错峰开学阶段, 但在线教学并没有结束, 也远 不会结束。经过疫期以来大规模的在线教学实践, 教育信 息化管理的优势充分展现出来, 这将推动全国高校教育信 息化管理水平在未来更上新台阶; 教学情境的改变为每位 教师创造了一次检视与反思自身教育理念、教学方式手段 方面的滞后与问题, 也引发了一场聚焦教学质量提升与教 学改革创新的热潮。“协作学习”模式不仅有利于改进线上 教学的弊端, 也为线下教学如何与线上教学相融合提供了 较好的实践思路, 但这种模式的具体组织形式、实施方式 与实施手段还有待更多经验总结, 其教学效果也有待进一 步验证。除此以外, 还有没有更多有助于线上线下相融合 的教学模式仍然值得继续探究, 线上课程的评价指标如何 与目前线下教学质量评估体系相整合也是亟待解决的助 力性问题。科学技术依然会日新月异, 将为教学创新带来 更多可能与机会, 但在“以人为本”的教育领域, 人工智能 永远无法替代教育者, 教育者与学习者永远是教育革命的 灵魂。

\section{参考文献}

１1］全国高等学校质量保障机构联盟（CIQA）：《疫情期间高 校教师线上教学调查报告》, 载邬大光微信公众号, https://mp.weixin.qq.com/s/eplOC9NpJKpXqqZCO3SD2A, 2020年4月 5 日; 《疫情期间大学生线上学习调查报告》, 载邬大光微信公众号, https://mp.weixin.qq.com/s/BN6o3qqUU0dJIksYQHxztw ， 2020年4月7日。

[2] [英] 杰里米. 诺克斯,肖俊洪. 慕课革命进展如何:慕课的三 大变化主题 [J]. 中国远程教育（综合版）,2018, 000(001):53-62。

[3] 汪琼,欧阳嘉煜,纪九梅,等.亚洲地区慕课发展五年回顾:从 2013年至2017年[J].中国远程教育,2019,000(004):54-59。 
[4] 卢强,郑晓旭,王艳.慕课发展路向审思:远程教育哲学视域[J] 现代远距离教育,2019,No.185(05):13-21。

[5] 资料源自2020年4月 10 日，教育部高等教育司司长吴岩在 “中国高校在线教学国际平台课程建设工作视频会议”上的 报告内容。

[6] 陈吉荣. 国外慕课研究最新发展述评 [J].外语教学与研究, 2016,048(001):118-127。

[7] [加]琳达·哈拉西姆, 肖俊洪译.协作学习理论与实践——在 线教 育质量的根本保证 [J]. 中国远程 教 育, 2015,000(008):5-16。

[8] Ki Mae Heussner, More Money for MOOCs: Coursera Nabs $\$ 43$ million from diverse set of Investors, (Jul 10, 2013),https://gigaom.com/2013/07/10/more-money-for-moocs -coursera-nabs-43m-from-diverse-set-of-investors/.

[9] 蒋志辉,赵呈领.学习者满意度:在线学习中教师支持服务的 终极归宿[J].现代远距离教育,2018,000(006):51-59。

[10] 何克抗.关于MOOCs的"热追捧"与"冷思考"[J].北京大学教 育评论, 2015, v.13;No.51(03):116-135+197。

[11] Laura Perna, et al., The Life Cycle of a Million MOOC Users, MOOC Research Initiative Conference, Austin, Texas, Dec 5,
2013 ,

https://www.mendeley.com/catalogue/b36d5a1f-6a67-3a55-b3 cb-65b4303f508e/.

[12] 王宇,罗淑芳,范逸洲,等.2017全球慕课发展回顾[J].中国远 程教育（综合版）,2018(9):53-61。

[13] 翁朱华.在线辅导:在线教学的关键一一访在线教学领域知 名学者吉利·西蒙博士 $[J]$.开放教育研究,2012,018(006):4-8。

[14] [美]尼尔·布彻,刘占荣,韩艳辉,等.网络学习质量指南(上) [J]. 中国远程教育,2013,000(007):62-64。

[15] 肖君,姜冰倩,陈海建.基于学习分析的体验式开放在线教学 环境研究--英国开放大学的实践与探索 [J]. 中国远程教育 （综合版）,2015,000(010):64-70。

[16］［美]尼尔·布彻(Neil Butcher),梅丽迪·威尔逊·斯其顿(Merridy Wilson-Strydom), 刘占荣(译者), 等.网络学习质量指南(下) [J]. 中国远程教育,2013(16):68-70。

[17] 周洲.在线教育课程质量评估研究[J].中国高等教育评估, 2017,000(002):19-23。

[18］《论语·卫灵公》。

[19]《礼记·学记》。 